\title{
Successful implementation of perioperative beta- blockade utilizing a multidisciplinary approach
}

\author{
[L'application réussie d'un bêta-blocage périopératoire par une approche \\ multidisciplinaire]
}

\author{
Samuel Armanious FFARCSI, ${ }^{*}$ David T. Wong MD, ${ }^{*}$ Edward Etchells FrCPC, $\dagger$ Patrick Higgins FFARCSI, \\ Frances Chung FRCPC*
}

\begin{abstract}
Purpose: To describe how we implemented a protocol for perioperative beta-blockade in patients with or at risk of coronary artery disease (CAD) undergoing major non-cardiac surgery and to present our results.
\end{abstract}

Methods: After institutional approval, from May 1999 to April 200I, patients with surgical and medical indications (CAD as indicated by previous myocardial infarction, typical angina or atypical angina with a positive stress test or at least two risk factors for CAD: age $65 \mathrm{yr}$, hypertension, smoking, high cholesterol, diabetes mellitus) for perioperative beta-blockade were identified preoperatively by anesthesiology and referred to the General Internal Medicine Service (MED). MED initiated patients on outpatient beta-blockers. The intraoperative anesthetic management was left to the discretion of the anesthesiologist. In the postanesthesia care unit (PACU), patients received iv metoprolol according to hemodynamic criteria. Postoperatively, patients were followed by MED for adverse cardiac events.

Results: Sixty-nine patients received perioperative beta-blockade. Preoperatively, $60 \%$ were started on metoprolol, $39 \%$ on atenolol and $1 \%$ on propranolol. In PACU, $42 \%, 9 \%$ and $38 \%$ of patients were given iv metoprolol 0, 5 and $10 \mathrm{mg}$ respectively. One patient was given glycopyrrolate in the PACU for bradycardia and none received vasoactive or inotropic agents. Three patients (4.3\%) had postoperative cardiac events.

Conclusions: With close collaboration between anesthesiologists, internists, PACU nurses and family physicians, a strategy for perioperative beta-blockade was implemented successfully in patients with cardiac risks. Beta-blockade was associated with few side effects and morbidities.

\begin{abstract}
Objectif : Décrire la mise en application d'un protocole de bêtablocage périopératoire chez des patients atteints, ou à risque, de coronaropathie (CP) devant subir une intervention non cardiaque majeure, et présenter nos résultats.
\end{abstract}

Méthode : Ayant reçu l'approbation de l'institution, les anesthésiologistes ont identifié, entre mai 1999 et avril 200 I, les patients admissibles à un bêta-blocage périopératoire selon des indications médicales et chirurgicales (CP révélée par un infarctus du myocarde antérieur, angine typique ou atypique démontrée par une épreuve d'effort positive ou au moins deux facteurs de risque de CP : âge $=65$ ans, hypertension, tabagisme, cholestérol élevé, diabète) et les ont été dirigés vers le General Internal Medicine Service (MED). Le MED a d'abord administré les bêta-bloqueurs en clinique externe. L'anesthésiologiste s'est chargé de la période peropératoire. À la salle de réveil (SDR), on a administré du métoprolol iv selon les critères hémodynamiques. Le MED a assuré un suivi postopératoire en raison de complications cardiaques possibles.

Résultats : Soixante-neuf patients ont reçu des bêta-bloqueurs périopératoires. Avant l'opération, $60 \%$ d'entre eux ont d'abord eu du métoprolol, 39 \% de l'aténolol et I \% du propranolol. À la SDR, 42 \%, $9 \%$ et $38 \%$ des patients ont respectivement reçu 0, 5 et $10 \mathrm{mg}$ de métoprolol iv. À la SDR, un patient a reçu du glycopyrrolate pour une bradycardie, mais aucun agent vasoactif ou inotrope n'a été donné. Trois patients (4,3\%) ont eu des problèmes cardiaques postopératoires.

Conclusion : La collaboration entre anesthésiologistes, internistes, personnel infirmier de la SDR et omnipraticiens a permis la mise en œuvre réussie d'une stratégie de bêta-blocage périopératoire chez des patients présentant des risques cardiaques. Peu de morbidité ou d'effets secondaires ont été associés au bêta-blocage.

From the Departments of Anesthesiology, ${ }^{*}$ and Division of General Internal Medicine and Clinical Epidemiology, $†$ Toronto Western Hospital, University Health Network, University of Toronto, Toronto, Ontario, Canada.

Address correspondence to: Dr. David T. Wong, Department of Anesthesia, EC 2-046, Toronto Western Hospital, University Health

Network, 399 Bathurst Street, Toronto, Ontario M5T 2S8, Canada. Phone: 416-603-5118; Fax: 416-603-6494;

E-mail: david.wong@uhn.on.ca

Supported by the Department of Anesthesiology, University Health Network, University of Toronto, Toronto, Ontario, Canada. Accepted for publication July 16, 2002.

Revision accepted November 6, 2002. 


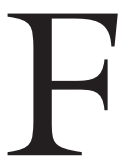

OR patients with or at risk of coronary artery disease undergoing major non-cardiac surgery, the most significant causes of mortality and cardiovascular morbidity are myocardial ischemia and myocardial infarction during the first week after surgery. ${ }^{1}$ Both myocardial ischemia and myocardial infarction increase the risk of serious cardiovascular morbidities..$^{2-4}$

During the perioperative period, the exaggerated sympathetic response that is associated with substantial increases in the heart rate (HR) throughout the hospitalization appear to be implicated in the occurrence of postoperative ischemic events. ${ }^{1}$ Several studies have shown the efficacy of perioperative beta-blockade in reducing the incidence of mortality and cardiovascular events in the perioperative period. Mangano et al. reported a randomized controlled trial of 200 patients with or at risk of coronary artery disease who underwent non-cardiac surgery. ${ }^{5}$ Overall mortality was significantly lower among the atenolol group than the placebo group for up to two years following hospital discharge. Wallace et al. ${ }^{6}$ concluded that perioperative administration of atenolol to patients with coronary disease significantly reduced the incidence of postoperative myocardial ischemia. Poldermans et al. showed that in high risk patients undergoing vascular surgery, perioperative bisoprolol reduced postoperative cardiac mortality and morbidity by $90 \%{ }^{7}$

Although many believe perioperative beta-blockade is indicated for patients at risk, there are several logistic difficulties in applying it to our patients. First, potential candidates for perioperative beta-blockade may be missed unless there is adequate screening preoperatively. Second, most anesthesiologists do not have the time or availability to initiate beta-blockade preoperatively and to follow-up patients postoperatively. Third, iv atenolol ${ }^{5}$ or bisoprolol ${ }^{7}$ is not available in Canada. The purposes of this study are to describe how we planned and implemented a practical protocol for perioperative use of beta-blockers in patients with or at risk of coronary artery disease undergoing major non-cardiac surgery in our institution, and to describe the results from our first 100 patients.

\section{Methods}

Preoperative management

The study protocol was approved by the Research Ethics Board, University Health Network. The implementation of perioperative beta-blocker strategy in our institution required close collaboration and team work between the anesthesiologist, the General Internal Medicine (MED) service, the family doctor, the surgeon, the postanesthesia care unit (PACU) nurses and the patient.
From May 1999 to April 2001, the identification of patients for perioperative beta-blockade started in the Anesthesia Preoperative Assessment Clinic. All candidates suitable for beta-blockers who fulfilled the inclusion criteria set by Mangano et al. ${ }^{5}$ (Appendix 1; available as "additional material" at www.cja-jca.org) were identified by the anesthesiologist and referred to the MED consult service. MED assessed patients' medical and surgical indications of perioperative beta-blockers and for any contraindication to beta-blockers. Patients on chronic beta-blockers were excluded from this perioperative beta-blockade protocol. MED ordered any further investigations to risk stratify patients before initiating preoperative beta-blocker therapy. Postoperatively, after these patients were discharged from the PACU, the MED service provided follow-up for patients on the wards until they were discharged home. MED ordered the required investigations for any patient who was suspected of any adverse event, e.g., angina, myocardial infarction, hypotension, congestive heart failure or ventricular dysrhythmia and they were responsible for necessary treatments.

Two information sheets were developed; one for the family physicians (Appendix 2; available as "additional material" at www.cja-jca.org) and one for the patients (Appendix 3; available as "additional material" at www.cja-jca.org). Patients were seen by the family physicians within one week of starting the beta-blockers, provided there was time prior to scheduled surgery, to assess any potential side effects from the new medication and for adjustment of the dose of beta-blocker to reach the target HR and blood pressure $(\mathrm{BP})$. Family physicians and patients received explanatory notes on the rationale and practicalities of perioperative beta-blockade.

On the day of surgery all patients were advised to take their oral beta-blockers. On arrival at the hospital, BP and HR of patients were measured and recorded in the chart.

\section{Intraoperative management}

The intraoperative anesthetic management, monitoring and other aspects of perioperative management were at the discretion of the attending anesthesiologist. There were no restrictions for postoperative analgesic management.

\section{Postoperative management}

\section{A) PACU}

The patient's BP and HR were monitored every five minutes by automatic BP cuff prior to and during the period of $i v$ metoprolol administration. 
PACU nurses administered iv metoprolol to patients according to specific guidelines, observed side effects such as hypotension, bradycardia, congestive heart failure, heart block or bronchospasm, and notified the anesthesiologist for appropriate management.

\section{Guidelines for administration of metoprolol infusion in the PACU}

The iv administration of metoprolol infusion required a systolic BP $100 \mathrm{mmHg}$ and HR 55 beats. $\mathrm{min}^{-1}$ at any time. If the initial PACU systolic BP was $<100$ $\mathrm{mmHg}$ or $\mathrm{HR}<55$ beats. $\mathrm{min}^{-1}$, no metoprolol was given. Otherwise, $5 \mathrm{mg}$ of metoprolol were infused over ten minutes. Five minutes after completion of the first dose of metoprolol, if the systolic BP was $>100$ $\mathrm{mmHg}$ and $\mathrm{HR}>55$ beats. $\mathrm{min}^{-1}$, another $5 \mathrm{mg}$ metoprolol were infused over ten minutes.

B) WARD

In the wards or intensive care unit, patients were followed-up by MED. Beta-blocker administration was continued providing BP and HR guidelines were met. Ordering of further investigations and clinical management were left to the discretion of MED service. Patients were assessed for the presence of any adverse cardiac events in the form of myocardial infarction, unstable angina, congestive heart failure or ventricular dysrhythmia as defined by standard criteria. ${ }^{6}$

\section{C) DISCHARGE}

Unless there were contraindications, patients were discharged home on beta-blockers to be followed-up by family physicians.

\section{Data collection}

All patients identified by anesthesiologists and accepted by MED for perioperative beta-blockers were recorded in a registry. Postoperatively, patient data from preoperative, intraoperative, PACU and ward phases were collected retrospectively by an anesthesiology fellow. Data were entered into a Microsoft Excel spreadsheet (Redmond, WA, USA) and subsequently analyzed by SPSS statistical software (Chicago, IL, USA). Continuous data are presented as mean \pm SD while categoric data are presented as numbers and percentages.

\section{Results}

From May 1999 to April 2001, 100 patients were identified by anesthesiologists and referred to MED for perioperative beta-blockade. Of the 100 patients, 31 were excluded by MED due to contraindications or other reasons: two had contraindications to betablockers, seven had unstable hemodynamics, 15 had
TABLE I data, inclusion criteria and surgical population

\begin{tabular}{ll}
\hline Patient population & \\
Age in yr (mean \pm SD) & $70 \pm 8$ \\
Range & $48-85$ \\
Sex males & 37 \\
$\quad$ females & 32 \\
& \\
Inclusion criteria & No of patients \\
Definite coronary artery disease & 30 \\
Previous myocardial infarction & 19 \\
Typical angina & 13 \\
Atypical angina & 2 \\
& \\
Risk factors for coronary artery disease & 39 \\
Age $\geq 65$ yr & 32 \\
Hypertension & 37 \\
Current smoking & 9 \\
High cholesterol & 12 \\
Diabetes mellitus & 12 \\
& \\
Types of surgery & \\
Vascular surgery & 15 \\
Neurosurgery & 5 \\
Orthopedic surgery & 36 \\
General surgery & 8 \\
Uro-genital surgery & 5 \\
\hline
\end{tabular}

low medical risk of developing cardiovascular morbidities (decided after reviewing further cardiac testing), three had low risk surgery and four were rejected for miscellaneous reasons, e.g., diabetes. Sixty-nine patients fulfilled the inclusion and exclusion criteria and were started on oral beta-blockers preoperatively.

Demographic data, inclusion criteria and surgical case-mix are shown in Table I. Cardiac investigations available at the time when patients were seen by MED and further tests ordered by MED are shown in Table II. The results of the newly ordered stress myocardial perfusion scans showed either no or limited reversibility. The only coronary angiogram ordered in a patient with discrepancy between clinical status and stress myocardial perfusion scan showed noncritical coronary artery disease. The results of a new cardiac investigation requested by MED did not affect the decision to proceed with the proposed non-cardiac surgery. None of these patients underwent prophylactic coronary angioplasty or coronary bypass surgery before the planned non-cardiac surgery.

Sixty-nine patients were started on beta-blockers in the preoperative period for a duration ranging between one and 45 days. The type and dosages of beta-blockers are shown in Table III. In the PACU, 29 (42\%) patients received $0 \mathrm{mg}$ metoprolol $i v$, six $(8.7 \%)$ 
TABLE II Preoperative cardiac investigations

\begin{tabular}{llll}
\hline Available at time of medical consultation & & Ordered by General Internal Medicine & None \\
\hline Stress electrocardiography & 10 & Stress electrocardiography & 7 \\
Stress myocardial perfusion imaging & 14 & Stress myocardial perfusion imaging & 1 \\
Coronary angiography & 6 & Coronary angiography & 6 \\
Echocardiography & 23 & Echocardiography & \\
\hline
\end{tabular}

TABLE III Preoperative oral beta-blockers dosage

\begin{tabular}{lccll}
\hline & $25 \mathrm{mg}$ & $50 \mathrm{mg}$ & $100 \mathrm{mg}$ & $\begin{array}{l}\text { Number } \\
\text { of patients }\end{array}$ \\
\hline $\begin{array}{l}\text { Metoprolol } \\
\text { (twice daily dosing) }\end{array}$ & 9 & 26 & 6 & 41 \\
$\begin{array}{l}\text { Atenolol } \\
\text { (once daily dosing) }\end{array}$ & 16 & 11 & 0 & 27 \\
$\begin{array}{l}\text { Propranolol } \\
\text { (four times daily dosing) }\end{array}$ & $40 \mathrm{mg} \cdot$ day $^{-1}$ & & 1 \\
\hline
\end{tabular}

received $5 \mathrm{mg}$ metoprolol while $26(37.7 \%)$ received 10 $\mathrm{mg}$ metoprolol. Eight patients (11.6\%) missed the PACU beta-blockers protocol due to omissions by anesthesiologists or the PACU nurses and did not receive any iv metoprolol in the PACU. Beta-blockade was continued on the wards for these patients.

Hemodynamic aberrations in the operating room or PACU were treated as required. Nineteen patients $(27.5 \%)$ required the administration of ephedrine, phenylepherine, atropine or glycopyrrolate. Except for one patient who needed glycopyrrolate $0.2 \mathrm{mg}$ in the PACU to counteract bradycardia, all drugs were given inside the operating room.

In the wards, two patients had episodes of hypotension which were responsive to fluid administration. Three patients $(4.3 \%)$ sustained a postoperative myocardial infarction during the first $48 \mathrm{hr}$ after surgery, which was treated medically. Two of these three myocardial infarctions were accompanied by congestive heart failure. One patient had a hypoxic episode secondary to atelectasis. All patients except one were discharged home on oral beta-blockers (44 on metoprolol and 24 on atenolol). The only patient whose beta-blocker was discontinued postoperatively had severe hypotension in the ward due to excessive blood loss.

\section{Discussion}

Perioperative beta-blockade in patients with or at risk of coronary artery disease was instituted successfully in our hospital. The majority of patients tolerated betablockade without difficulties and there were few adverse events.

Of the 100 patients referred to MED, 31 were excluded for various reasons. Anesthesiologists used inclusion criteria outlined by Mangano et al. ${ }^{5}$ The most common reason for exclusion by MED was determination of a low cardiac risk after results of additional cardiac investigations were known. It was felt that perioperative beta-blockade would not benefit patients with low cardiac risks. ${ }^{8}$

The most common indications for perioperative beta-blockade were age $65 \mathrm{yr}$ and hypertension. Fiftytwo percent of patients were from orthopedic surgery. Mangano's ${ }^{5}$ surgical population consisted of a balanced mixture of major vascular, intra-abdominal, orthopedic, neurosurgical and other surgeries. Our institution has clinical foci on neurosurgical, neurovascular and orthopedic cases and our study population reflected the predominance of such patients.

Nineteen percent of the patients needed further cardiac investigations in the form of echocardiography, stress electrocardiography/myocardial perfusion imaging or coronary angiography. However, the results of the stress myocardial perfusion scans and coronary angiography did not affect the decision to proceed with the scheduled surgery or result in coronary revascularization before the non-cardiac surgery. Current evidence suggests that preoperative prophylactic coronary revascularization is rarely indicated except in the presence of independent indications for coronary revascularization. ${ }^{8,9}$

Our patients were started on oral beta-blockers one to 45 days before surgery. Our protocol is different from Mangano's ${ }^{5}$ where in beta-blockers were started $30 \mathrm{~min}$ before patients entered the operating room. Our goal was to have the patients in a steady state of beta-blockade at the time of surgery. This may explain that $42 \%$ of our patients did not require any iv metoprolol and $8 \%$ of patients received a half dose $(5 \mathrm{mg})$ 
of metoprolol in the PACU. In contrast, in Mangano's ${ }^{5}$ study, $74 \%$ of subjects received a full dose, $10 \%$ received a half dose while $15 \%$ did not receive iv beta-blockers in the PACU.

Provision of preoperative outpatient beta-blockade has several potential advantages: a stable hemodynamic state, dosage titration preoperatively, detection of adverse effects to beta-blockers and allowing MED to know the patient before date of surgery. For institutions with adequate MED support, the above is the recommended approach ${ }^{9}$ and has worked well in our hospital. However, for institutions without the necessary MED support, administration of preoperative beta-blockers immediately before surgery may be the only option. Regarding postoperative follow-up and titration of beta-blockers, MED support is essential as most anesthesiologists do not have the time or availability to care for patients on the ward.

In Mangano's study ${ }^{5}, i v$ atenolol was administered in the PACU. One impediment to implementation of perioperative beta-blockade is that iv atenolol is not available in Canada. After review of the literature ${ }^{10-13}$ and consultation with the hospital Pharmacy and Therapeutics Committee, a decision was made to use equipotent doses of $i v$ metoprolol $(0-10 \mathrm{mg})$ in place of atenolol $(0-10 \mathrm{mg})$ for PACU beta-blocker administration. The PACU nurses, previously not certified to give $i v$ metoprolol, were trained regarding the criteria and method for $i v$ metoprolol administration and observation for side effects e.g., hypotension, bradycardia, congestive heart failure, heart block or bronchospasm. This allowed them to give iv metoprolol under the indirect supervision of anesthesiologists.

In previous perioperative beta-blockers studies, oral beta-blockers were continued for seven days ${ }^{5,6}$ or 30 days postoperatively. ${ }^{7}$ All but one of our patients were discharged on oral beta-blockers. Current literature suggests that long-term beta-blockade in patients with coronary artery disease improves long-term outcome. ${ }^{14}$ Discharging patients home on chronic beta-blockers appears reasonable in this context.

Nineteen patients required vasoactive drugs to maintain hemodynamics. The majority of patients were elderly with co-morbidities undergoing major non-cardiac surgery with substantial blood losses. In addition, many patients received spinal or epidural anesthesia. Therefore, the incidence of perioperative vasoactive drug usage was not surprising.

There was no mortality in our patient population. The incidence of adverse cardiac events was $4.3 \%$ and consisted of nonfatal myocardial infarction with or without congestive heart failure. A recent meta-analysis $^{15}$ consisting of 1,994 cases undergoing risk stratifica- tion before vascular surgery showed postoperative adverse cardiac event rates from $2.6 \%$ to $21.7 \%$ with a mean of $11.5 \%$. Our low cardiac event rate may be attributed to a different risk population or our nonsystematic search for postoperative cardiac events.

There are several limitations of this study. First, despite our best attempts to implement perioperative beta- blockade in enrolled patients, eight still did not receive iv metoprolol as per protocol. We have since instituted several measures including putting a "betablocker protocol" label on the operating room list and putting a colour sticker indicating "beta-blocker protocol" on the anesthetic record to avoid protocol failures. Second, this is a retrospective cohort study intended to describe our experience at implementing a program of perioperative beta-blockade. It is not intended to demonstrate the efficacy of beta-blockade in reducing perioperative morbidity and mortality which has been shown elsewhere. ${ }^{5-7}$ Third, the ordering of investigations to confirm postoperative adverse cardiac events was left to the discretion of the MED team. Therefore, we may have underestimated the true incidence of adverse cardiac events.

We have shown that perioperative beta-blockade can be implemented successfully by a multidisciplinary approach with a defined protocol, vigilant screening at the anesthesia preoperative assessment clinic, cooperation of anesthesiology and MED, training of the PACU nurses, and education of family physicians and patients. Patients were initiated on beta-blockers on an outpatient basis and discharged home on betablockers unless contraindicated. There were very few side-effects from beta-blockers and infrequent perioperative morbidities.

\section{Acknowledgements}

We wish to thank Anne Mullin, Anesthesia consult clinic secretary for her invaluable help in identification and compilation of perioperative beta-blocker candidates; and Christine Drane, Anesthesia secretary for her excellent help in preparing this manuscript.

\section{References}

1 Mangano DT, Wong MG, London MJ, Tubau JF, Rapp $J A$. Perioperative myocardial ischemia in patients undergoing noncardiac surgery-II: incidence and severity during the $1^{\text {st }}$ week after surgery. The Study of Perioperative Ischemia (SPI) Research Group. J Am Coll Cardiol 1991; 17: 851-7.

2 Mangano DT, Browner WS, Hollenberg M, London MJ, Tubau TF, Tateo IM. Association of perioperative myocardial ischemia with cardiac morbidity and mortality in men undergoing noncardiac surgery. The 
Study of Perioperative Ischemia Research Group. N Engl J Med 1990; 323: 1781-8.

3 Mangano DT, Browner WS, Hollenberg M, Li J, Tatoe $I M$. Long-term cardiac prognosis following noncardiac surgery. JAMA 1992; 268: 233-9.

4 Browner WS, Li J, Mangano DT. In hospital and longterm mortality in male veterans following noncardiac surgery. The Study of Perioperative Ischemia Research Group. JAMA 1992; 268: 228-32.

5 Mangano DT, Layug EL, Wallace A, Tateo I. Effect of atenolol on mortality and cardiovascular morbidity after noncardiac surgery. N Engl J Med 1996; 335 : 1713-20.

6 Wallace A, Layng B, Tatoe I, et al. Prophylactic atenolol reduces postoperative myocardial ischemia. The McSPI Research Group. Anesthesiology 1998; 88: 7-17.

7 Poldermans D, Boersma E, Bax JJ, et al. The effect of bisoprolol on perioperative mortality and myocardial infarction in high-risk patients undergoing vascular surgery. Dutch Echocardiographic Cardiac Risk Evaluation Applying Stress Echocardiography Study Group. N Engl J Med 1999; 341: 1789-94.

8 Fleisher LA, Eagle KA. Lowering cardiac risk in noncardiac surgery. N Eng J Med 2001; 345: 1677-82.

9 Eagle KA, Berger PB, Calkins H, et al. ACC/AHA Guideline Update for Perioperative Cardiovascular Evaluation for Noncardiac Surgery-Executive Summary. A report of the American College of Cardiology/American Heart Association Task Force on Practice Guidelines (Committee to Update the 1996 Guidelines on Perioperative Cardiovascular Evaluation for Noncardiac Surgery). Circulation 2002; 105 : 1257-67.

10 ISIS-I (First International Study of Infarct Survival) Collaborative Group. Randomized trial of intravenous atenolol among 16027 cases of suspected acute myocardial infarction: ISIS-I. Lancet 1986; 2: 57-66.

11 ISIS-I (First International Study of Infarct Survival) Collaborative Group. Mechanisms for the early mortality reduction produced by beta-blockade started early in acute myocardial infarction: ISIS-I. Lancet 1988; 1 : 921-3.

12 Anonymous. Metoprolol in acute myocardial infarction. Patients and methods. The MIAMI Trial Research Group. Am J Cardiol 1985; 56: 3G-9G.

13 Anonymous. Metoprolol in acute myocardial infarction (MIAMI). A randomised placebo-controlled internaltional trial. The MIAMI Trial Research Group. Eur Heart J 1985; 6: 199-226.

14 Gibbons RJ, Chatterjee K, Daley J, et al. ACC/AHA/ACP-ASIM guidelines for the management of patients with chronic stable angina: a report of the American College of Cardiology/American Heart
Association Task Force on Practice Guidelines (Committee on Management of Patients With Chronic Stable Angina). J Am Coll Cardiol 1999; 33: 2092-197.

15 Shaw LJ, Eagle KA, Gersh BJ, Miller DD. Meta-analysis of intravenous dipyridamole-thallium-201 imaging (1984 to 1994) and dobutamine echocardiography (1991 to 1994) for risk stratification before vascular surgery. J Am Coll Cardiol 1996; 27: 787-98. 